\title{
El cambio en los procesos de mediación sígnica. Una lectura peirceana de la evolución en las mediaciones
}

\author{
The change in the processes of signs mediation. A peirceanian reading on \\ mediations' evolution
}

\author{
Paulo Damián ANICETO \\ Universidad Nacional de Córdoba - Argentina \\ paulodamiananiceto@gmail.com
}

Cómo citar este artículo: ANICETO, Paulo Damián (2015): «El cambio en los procesos de mediación sígnica. Una lectura peirceana de la evolución en las mediaciones», Mediaciones Sociales, no 14, pp. 13-39. DOI: http://dx.doi.org/10.5209/rev_MESO.2015.n14.51557

Recibido: 9 de diciembre de 2014.

Aceptado: 28 de octubre de 2015.

\section{RESUMEN}

En este ensayo, propongo un relevo crítico del estado de los debates sobre las funciones de normalización y transformación desplegadas en los procesos de mediación. El ámbito de tales debates, donde inscribo este mismo relevo, es el de la pragmática peirceana, que ha ofrecido, y ofrece, un escenario de divergencias en torno a un tema específico: la comunidad de pensamiento como normalización última del conocimiento generado en los sujetos. Estas páginas desarrollan una descripción de los posicionamientos en querella y proponen, finalmente, una comprensión alternativa que entiendo superadora.

PALABRAS CLAVE: cambio, colectivos de identificación, evento-signo, mediación sígnica, normalización.

\section{ABSTRACT}

In this paper, I propose a critical relief of state of discussions on normalization and transformation function deployed in the mediation process. The scope of such, where I enroll this relay, is the peirceanian pragmatism, which has offered, and offers today, a stage of discrepancies on a specific subject: community of thought as last normalization of the knowledge generated in subject. These 
pages develop a description of positions in dispute and propose, finally, a alternative approach I think it's resolutive.

KEYWORDS: change, collectives of identification, event-sign, mediation of signs; normalization.

Sumario: 1. Introducción. 2. La mediación en el crecimiento del sistema de signos. 3. Comunidad de pensamiento vs. doble racionalidad de la acción social. 3.1. El espacio semiótico común y las conciencias. 3.2. El constructivimo y el no-constructivismo. 3.3. Los marcos comunitarios y la Terceridad. 4. La ley y la verdad. 4.1. Regímenes de enunciación. 4.2. La verdad en su postergación. 5. Las palabras y las cosas. 5.1. El cambio en el sistema de la lengua. 5.2. El cambio en el discurso. 6. Conclusión. Bibliografía.

"Such is everything which is essentially a Sign-not the mere body of the Sign, which is not essentially such, but, so to speak, the Sign's Soul, which has its Being in its power of serving as intermediary».

C. Peirce (1908; CP 6.455)

\section{INTRODUCCIÓN}

Actualmente, asistimos a la multiplicación de los ámbitos de debate sobre el fenómeno de la mediación. Tanto desde perspectivas cercanas al cognitivismo y teorías de la representación como desde numerosos estudios provenientes del campo de la mediatización, el objeto en cuestión se ha diseminado al punto de conformar un todo pluridimensional que exige una re-sistematización.

En estas páginas abordamos problemáticas específicas de la mediación proponiendo una clave semiótica de lectura. Reconocemos en la teoría peirceana de los signos un albergue principal de temas que suscitan la atención de estos debates. Albergue que consideramos necesario revisitar para otorgar claridad y sistemática al caudal de producción conceptual.

El pragmaticismo de Peirce ha sido tratado con diverso rigor. Los exponentes actuales del peirceanismo con más resonancia son Ransdell (1997), Barrena (2006), Debrock (1991), da Silveira (2004), Colaprieto (1995) y Andacht (2008). Cada uno ha asumido los supuestos peirceanos sobre la mediación y la finalidad con distintas miradas, provenientes todas ellas de dos principales corrientes: el constructivismo y el no-constructivismo.

Aquí los retomamos otorgando un lugar central a la función de mediación sígnica, denominada por el mismo Peirce (\& Barrena, 1997; CP 6.455, 1908) Alma de los signos. Nos proponemos comprender el fenómeno del crecimiento de los signos y po- 
nerlo en relación con la noción peirceana de la mediación. Con este objetivo nos ubicamos en la intersección entre la teoría de la mediación y la teoría de los signos, observando especialmente que el lugar asignado por Martín Serrano (2008) a la categoría modelos de la mediación no clausura nuevas reflexiones a la luz de los aportes del peirceanismo.

\section{LA MEDIACIÓN EN EL CRECIMIENTO DEL SISTEMA DE SIGNOS}

Peirce atribuye a los signos la función de mediación de la experiencia. El autor lo plantea en términos abstractivos: el primer acercamiento al objeto es el que se produce con la observación de los Universos de Experiencia (Peirce \& Barrena, 1997; CP $6.452,1908)$. El signo producido, denominado representamen, asume una relación triádica: con un fundamento, un objeto y un interpretante (Peirce \& Restrepo, 2003; CP 2.229, c.1897). Es decir, el representamen es un signo que está por algo (relación con su objeto), representando algunos de sus respectos (relación con el fundamento), para generar en la mente de alguien más un signo más desarrollado sobre el mismo objeto (relación con su interpretante). En otras palabras, el pragmatismo peirceano plantea que la función mediadora de los signos está asociada constitutivamente con el crecimiento del conocimiento de un objeto.

La experiencia albergada en la mente, pasible de asociarse a un objeto del mundo, constituye la denominada Primeridad en el proceso de crecimiento de los signos. La Segundidad, denomina ya una relación de contigüidad o conexión existencial entre la experiencia y el mundo. El proceso de sentido orientado en esta dirección positiva genera en la mentalidad de alguien más una nueva relación signo-objeto. En este punto se produce una relación simbólica por la que una Terceridad evalúa y sanciona la relación Segunda entre un signo y el mundo observado. Esta otra mentalidad, afirma Peirce, representa el criterio científico comunitariamente consolidado que, al evaluar la relación sígnica, se convierte a su vez en un signo a ser evaluable por otra Terceridad, también generada en el perímetro de una comunidad de pensamiento. La "observación de los Universos de Experiencia" a la que hace referencia nuestro autor es atribuida a un criticismo moderado históricamente por leyes comunitarias.

Estructuramos estas páginas en dos grandes secciones. La primera se propone presentar una querella abierta en el ámbito del pragmatismo peirceano actual. La discusión se ha entablado por definir el lugar que debe asignársele al cambio o a su posibilidad en los procesos de mediación. Esto abre un espectro de temas, dentro del cual nos limitaremos a abordar aquellos que creemos de mayor peso específico: las nociones de conciencia en disputa y los vínculos entre las representaciones individuales y los marcos comunitarios de interpretación. La segunda sección se interesa por ligar las problemáticas anteriores a la comprensión necesaria de una específica forma 
de construcción de verdad y al papel desempeñado por la ley de la comunidad en esa construcción.

\section{COMUNIDAD DE PENSAMIENTO VS. DOBLE RACIONALIDAD DE LA ACCIÓN SOCIAL}

Como dijimos, una conciencia científica que mediatiza el conocimiento del mundo es constituida en un medio ambiente comunitario. La noción peirceana de comunidad científica supone suspender la distinción serreana de la doble racionalidad de la acción social (Martín Serrano, 2008). Este debate inserta el primer aspecto que deseamos tratar aquí: las posibilidades del cambio en los marcos comunitarios.

Si entendemos las representaciones individuales como signos normalizados por una ley comunitaria, no es posible distinguir una racionalidad privada (del uso funcional) de una lógica histórica comunitaria, toda vez que la primera es formada y moldeada al calor del desarrollo progresivo de la segunda. Bajo el prisma teórico peirceano, la racionalidad que Martín Serrano (2008: 76) denomina de uso funcional (individual) es entendida como una habilidad perceptual construida en la pertenencia a una comunidad. Se constituye, por lo tanto, en su expresión actualizada en el contexto de la comunicación. Es este el punto de partida para reflexionar sobre la transformación en los procesos de mediación.

La Terceridad que normaliza las representaciones y así les otorga significado es generada en comunidad. Revirtiendo los términos de Martín Serrano (2008: 76) los agentes sociales que toman en cuenta la necesidad histórica comunitaria confluyen en un espacio semiótico común.

\subsection{El espacio semiótico común y las conciencias}

Ahora bien, ese espacio semiótico, según el post formalista ruso Mijaíl Bajtín (1982) es el lugar de formación de las conciencias individuales que, en la comunicación discursiva, se reservarán la posibilidad de la divergencia y la creación. El peirceanismo no-constructivista entiende los modelos de la mediación, en contraste, como especiales matrices de representación incorporadas por un sujeto que no detenta el poder último de la creación y el cambio.

Ambas posturas, el constructivismo y el no-constructivismo, expresan dos interpretaciones peirceanas de la función mediadora de la representación y las posibilidades del cambio.

El debate referido a la eficacia normalizadora de los marcos comunitarios y la función de mediación de los signos constituye la problemática que abordamos a continuación. 


\subsubsection{Signo como representación y objeto a ser representado}

Debemos introducir a la concepción peirceana de mediación sígnica, precedente de los posicionamientos en controversia. Según Peirce (\& Restrepo, 2003; CP 2.228, c.1897), el sujeto piensa en los objetos del mundo en un instante irrepetible del tiempo. Así, el conocimiento mediado de tales objetos podrá crecer a condición de que existan futuras representaciones. De aquí extraemos dos cosas. En primer lugar, ni la experiencia del mundo ni el mundo mismo existen como tales sin su mediación. No hay cosa alguna que sea en sí misma. En segundo lugar, dado que las mediaciones son producidas en marcos comunitarios normativos, su propósito es el de "descubrir lo que debe ser y no meramente lo que es en el mundo real" (Peirce \& Restrepo, 2003; CP 2.228, c.1897). En este sentido, todo lo real es real por ser relativo a la mediación de la mente ${ }^{1}$. Basta, para comprenderlo, la ilustrativa afirmación de Eliseo Verón (2001: 14): "la mediatización de la sociedad industrial mediática hace estallar la frontera entre lo real de la sociedad y sus representaciones".

Los sentidos, asegura Peirce (CP 5.264-317; Peirce \& Vericat, 1988), son mecanismos de abstracción. El encuentro con lo real no-representado es mediado, desde un comienzo, por los sentidos, herramientas de la percepción. Ahora bien, y este es el aspecto que interesa a nuestra reflexión, lo percibido es representado por inferencias que serán re-interpretadas por nuevas inferencias: "hipótesis para cuya resolución se requieren nuevas hipótesis" (Peirce \& Vericat, 1988; CP 5.303, 1868).

En otras palabras, los modelos de mediación del mundo no son sólo incorporados por el sujeto que elabora las representaciones, sino reeditados por él mismo con un criterio adquirido en su pertenencia comunitaria. Sara Barrena (\& Peirce, 1997; CP $6.452-485,1908)$ valora este aspecto al asegurar que el pragmatismo de Peirce establece una continuidad entre experiencia y teoría, y entre teoría y práctica.

\subsubsection{El cambio y la continuidad de la conciencia}

En este sentido, el conocimiento mediado de los objetos del mundo, por ser un proceso en crecimiento, no puede ser alcanzado en un instante de cognición de la mente, sino que es el producto de la continuidad de nuestra conciencia. Esta continuidad, que asegura el conocimiento del mundo, es posibilitada por lo que Peirce (\& Vericat, 1988; CP 5.264-317, 1868) denomina fuerza efectiva real, subyacente a la conciencia misma. Este es uno de los principales elementos de la lógica peirceana.

\footnotetext{
${ }^{1}$ Vale tener presente, para avanzar sopesando las consecuencias, una advertencia de Barrena y Nubiola (2006) sobre el concepto de mente/mentalidad en Peirce. La autora nos recuerda que el conocimiento es un proceso comunicativo y que, por lo tanto, la mente no es algo atrapado en cada persona, sino un fenómeno externo.
} 
Ubica a la mediación en la sucesión y en el crecimiento de matrices normativas sociales que determinan el deber ser de la representación.

Umberto Eco (1992) nos indica el carácter positivo de esta determinación cuando considera que Peirce entiende la comunidad como garante intersubjetiva de la verdad del mundo. Lo verdadero de lo que se encuentra por conocer es aquello que conecta directamente nuestras cogniciones con un conjunto de conocimientos preexistentes de una comunidad que nos contiene. La comunidad aprueba una cierta forma arbitraria de relacionar, por remisión, las palabras y los objetos (Peirce \& Barrena, 2005; CS, 2.274-308, 1903). La ley y lo universal son, con todo, aspectos que subyacen al acto de mediación de la conciencia, sus componentes constitutivos.

La comunidad opera como un principio trascendental de la mediación sígnica, más allá de las intenciones individuales del intérprete concreto (Eco, 1992). El individuo que cree decir el mundo no lo percibe sino a través de habilidades perceptuales adquiridas en una comunidad de límites poco claros. Este es el campo en el que se inscriben los debates en torno a los márgenes de la creatividad individual en los procesos colectivos de sentido.

A esta altura, hemos ofrecido distintas lecturas de la función mediadora de los signos definida por Peirce. Si lo hicimos de manera acumulativa y recurrente ha sido para poner de relieve que un análisis de la relación entre la Terceridad y la mediación debe tener en cuenta que las leyes comunitarias solidificadas históricamente no necesitan sancionar las representaciones de los individuos a cada momento. Las habilidades perceptuales (CP 5. 93-119; Peirce \& Negro Pavón, 1978) funcionan como los definidos por Piaget (2000) esquemas de asimilación: la actividad cognitiva se desarrolla y estructura a partir de su confrontación con problemas de su medio. Lo que habita en los sujetos de conocimiento es un esquema asimilado, definido por Peirce, como vimos, criticismo moderado (Peirce \& Barrena, 1997; CP 6.452-485, 1908).

\subsection{El constructivismo y el no-constructivismo}

La conformación de un prisma peirceano de lectura de los modelos de la mediación permitirá, por lo dicho, comprender la relación simbólica de normalización que se establece entre comunidad e individuo en los procesos de producción de mediaciones. Con ese propósito describimos las dos corrientes del peirceanismo nombradas, constructivista y no-constructivista, implicadas en el debate por definirla eficacia normalizadora que la comunidad conserva en los procesos de mediación. ¿Qué posibilidades existen de pensar en un sujeto creativo agente del cambio e interviniente en los procesos de conocimiento del mundo? ¿El potencial creativo es reserva de un 
agente social o de procesos colectivos de transformación? La respuesta que encontramos en el ámbito actual de estudios peirceanos no es unívoca.

\subsubsection{Enfoque no-constructivista}

La primera y principal diferencia entre ambas posturas reside en las interpretaciones del papel de la conciencia individual en la función de la mediación. El noconstructivismo asume la concepción de un sujeto descentrado e integrado a una comunidad de pensamiento con límites poco claros. La realidad es entendida como realidad producida e implica en última instancia la presunción de una comunidad. Guy Debrok (1991: 56), en esta dirección, afirma que "el contexto completo indica que la mente misma ha sido producida por representaciones naturales". La concepción de Debrok sobre la mente toma distancia de aquella que defiende la existencia de voluntades individuales innovadoras. Compone, en cambio, la idea de una inteligencia colectiva que normaliza el flujo de signos de mediación con objetivos de largo plazo.

En la misma dirección es formulada la propuesta del norteamericano Michael Shapiro (2002). En Aspects of a Neo-Peircean Linguistics, el autor recupera la noción de deriva introducida por Edward Sapir al sostener que "la deriva de un lenguaje es constituida por selecciones inconscientes" (Shapiro, 2002: 112).

Advertiremos que el no-constructivismo no concibe un sujeto inconsciente, sino una conciencia individual orientada en el ejido de una mentalidad comunitaria e históricamente constituida. Esta sede es la misma en la que Debrock (1991) asienta su propuesta, al asegurar que "la información lingüística siempre contiene el germen de la ambigüedad, de interpretaciones diferentes" y donde Ransdell (en Andacht, 2003: 5) afirma que "las convenciones son regularidades en el comportamiento mantenidas por un interés en la coordinación y por una expectativa de que los otros cumplirán con su parte". En este marco, no es posible postular la autonomía individual, una voluntad subjetiva desprendida del desarrollo del proceso de mediación sígnica. No es dable pensar en un sujeto que decide conscientemente por sí mismo, porque el anclaje último de la mediación no está en él, sino en el carácter colectivo de las habilidades aprendidas. Shapiro (2002) es taxativo: el poder de innovación de la conciencia individual en el flujo de signos nació un error de categoría.

\subsubsection{Enfoque constructivista}

La lectura que atribuye autonomía a la voluntad individual en la producción de símbolos que mediarán los objetos del mundo asume la existencia de causas. Esto es: el avance de la cadena de mediaciones sígnicas vendría dado por la acción voluntaria 
de innovación y creatividad. El enfoque constructivista, ligado a la psicología del desenvolvimiento, distancia la formulación de sus principios elementales de las lecturas peirceanas no-constructivistas. En palabras de Fernando Andacht (2013: 24),

"la voz del influyente psicólogo/filosofo del lenguaje ruso Vigotski, no propone aquí otra forma de pensar nuestros signos, una que es aliada a la consciencia, a la subjetividad, un camino que la lógica antipsicologista (pero no antipsicológica) de Peirce evita para construir su teoría de la semiosis, de la acción de los signos autónoma y teleológica".

La línea constructivista, que podemos llamar, con Andacht, psicologista, se apoya sobre una afirmación: el conocimiento mediado del mundo es una "expresión evolucionada de una comunidad que camina en vista de lo que se le ofrece como objeto de deseo y realización" (da Silveira, 2004: 76). Esta afirmación encierra la suposición de que el sujeto individual, a través del conocimiento mediado del mundo, sería capaz de reconocer sus principales objetivos o una hoja de ruta como elementos de sus propias representaciones.

Esta perspectiva se basa en la creencia en subjetividades que discriminan las reglas de su comunidad de pensamiento sin delegar su participación activa, consciente e instituyente.

En la misma línea se inscriben los estudios de Vincent Colapietro (1995: 482). El autor fundaba su visión en la percepción de que "Peirce claramente asumió la realidad de la inconciencia" y de que no habría explorado sustancialmente esta dimensión de la mente. Colapietro (1995: 482) propone que las nociones del pragmatismo de Peirce "podrían ser usadas para articular una aproximación peirceana distintiva a la inconsciencia". El espacio simbólico emplazado como un espacio del agente del cambio no había sido considerado por el lógico norteamericano. Su planteamiento considera, en definitiva, que es posible desarrollar un abordaje semiótico de la inconsciencia y comparar esta aproximación con las realizadas por Sigmund Freud, Carl Jung, Jacques Lacan, Julia Kristeva y otros.

La masa abundante de cualidades del mundo pasibles de ser mediadas constituye una totalidad en potencia. El enfoque constructivista entiende que, en relación con esa potencialidad, las representaciones subjetivas son efectos de control de una actividad mental individual. La perspectiva no-constructivista entiende, en cambio, que la mediación sígnica emergente obedece a la actividad de una percepción inmediata (Ransdell en Andacht, 2003), entrenada el seno de una comunidad y punzada por la fuerza ciega de los fenómenos reales (fuerza efectiva real).

En función de lo dicho es posible recomponer la principal evidencia de la línea divisoria entre las perspectivas peirceanas constructivista y no-constructista. El argumento constructivista se esgrime a favor de las causas y se constituye, por esto, la 
hipótesis de que existiría la posibilidad de arribar a la primera de las causas, independiente y autónoma de la primera mediación. La variante no-constructivista, en oposición, comprende que la representación de algunas cualidades del objeto no se instaura como causa de futuras representaciones. Es generada, en cambio, como un evento de signo (Debrock, 1991). Éste produce una diferencia que no remite a una cualidad absoluta, primera, sino que provoca efectos prácticos en un proceso complejo de conocimiento. En esta línea, Shapiro (2002: 122) asegura que "Peirce entendió una causa final como siendo una posibilidad, que tiene una tendencia a convertirse en actual" (el subrayado es mío).

\subsection{Los marcos comunitarios y la Terceridad}

Peirce denomina "Terceridad" (Peirce \& Barrena, 1997; CP 6.452-485) a una dimensión de las mediaciones sígnicas. La Terceridad de una mediación es el componente que aplica sobre otras mediaciones una serie de normas comunitarias y generales. Siguiendo este razonamiento, si decimos que todas las mediaciones se encuentran normalizadas, también debemos agregar que tienen la cualidad de ser normalizadoras.

Ahora bien, ¿̇es posible pensar en la mediación normalizadora como un impedimento del cambio? Los exponentes del constructivismo sostienen que no, y algunos de los representantes del no-constructivismo hasta ignoran la importancia que encierra esta pregunta. Aquí daremos un relieve diferencial a la cuestión, como aporte al debate sobre los procesos de cambio social operado en las mediaciones de la cultura.

\subsubsection{Ley para el no-constructivismo}

Uno de los argumentos sobre la normalización de las representaciones es articulado por el no-constructivismo, con algunas contradicciones internas al mismo enfoque. Una es la ofrecida por el norteamericano Joseph Ransdell y otra, la del uruguayo Fernando Andacht, donde aquella hace eco y por la cual resuena en el ámbito hispanohablante de estudios peirceanos. Ambas representan la controversia interna del planteamiento no-constructivista. Elaborar una reseña de tal controversia, conducirá a pensar en las relaciones posibles entre la función de mediación sígnica y el cambio.

Por su parte, Joseph Ransdell (1997: 12) señalaba, en un texto de fines del siglo pasado, lo que entendía como el núcleo crítico de la discusión:

"estamos acostumbrados a pensar que, como intérpretes y usuarios de los signos, los tenemos en nuestro poder para dar significados a las cosas por puro acto de voluntad e intención (...) hablamos sobre inventar lenguajes que de alguna manera serán libres de 
las limitaciones inherentes en las palabras que usamos en el proceso de invención de esas cosas nuevas".

El aspecto ponderado por Ransdell (1997) constituye, hoy, el eje organizador de los debates sobre la mediación: las formas de la innovación y la posibilidad de cambio. Tiempo después, el mismo Ransdell (en Andacht, 2003: 234), al referirse a los efectos de la tecnología de la mediación, consideraría que la mejor manera de educar en el consumo de la imagen televisiva es "volverla disponible y entrenar a mis hijas en cómo lidiar con ella de modo inteligente". Este planteamiento encierra una dimensión simbólica que es posible contestar desde la teoría de Peirce. El lógico norteamericano afirmaría, en contraste, que una mentalidad que entrena habilidades de consumo televisivo ha sido históricamente formada en torno de una economía occidental de percepción. Una mentalidad con preferencias aprendidas, sometida a un régimen que entiende singularmente los conceptos libertad y regulación. Con todo, entrenar una inteligencia que interprete las representaciones visuales disponibles (como propone Ransdell) no es menos restrictivo que desprenderse de un televisor.

En la misma dirección, y en contrapunto con la idea expresada por él mismo en su fragmento anterior, Ransdell (en Andacht, 2003) alude a espíritus mezquinos que juzgan las mediaciones potenciales como peligrosas. Especialmente "en países aun fuertemente influidos por la iglesia" (en Andacht, 2003: 230).

Siguiendo el pragmaticismo de Peirce (\& Barrena, 1997; CP 6.476), lo denominación espíritu mezquino podrá designar una racionalidad tanto conservadora como revolucionaria, pero siempre normalizadora. Siempre esquiva a los peligros aparejados en el abanico de mediaciones posibles. Si sólo una parcialidad de ellas se hace efectiva, es por la acción de una ley de una comunidad de pensamiento.

En el sistema conceptual peirceano, la mediación que normaliza (la Terceridad) mediaciones anteriores toma la forma de una nueva mediación dando continuidad al proceso de sentido en esa dirección positiva. Es decir, las mediaciones despliegan una función paradójica: hacen avanzar el conocimiento del objeto, pero a través de evaluarlo y reorientarlo. Por lo tanto, la hipótesis que atribuye a la religión y a formas de conservadurismo la represión de la potencia creativa de las mediaciones es contestable: siempre se espera la llegada de una Terceridad, de la Iglesia o del Anarquismo, del Comunismo o del más flexibilizado Liberalismo.

De forma análoga el uso que Fernando Andacht $(2003,2004)$ de la categoría tecnocinismo, acuñada por el alemán Horst Bredekamp (en Andacht, 2003). El uruguayo le da una función en al reflexionar sobre la comunicación masiva a través de imágenes tecnológicas. Si bien reconocemos el interés de la discusión en un contexto de expansión tecnológica y sus efectos en los procesos de significación, es posible sopesar críticamente sus implicancias teóricas. El tecnocinismo describe una posición que, 
en palabras de Andacht (2003: 223-224) "concibe los signos icónicos como temibles y peligrosos señuelos diseñados para atrapar la versión moderna del rústico o del ingenuo".

Es iluminado aquí una contramarcha interna de la perspectiva no-constructivista, en la que ambos autores participan. En la entrevista que Andacht (2003: 227) realizó a Ransdell, el norteamericano se refiere al tema en los mismos términos, al considerar que algunos

\footnotetext{
"tienden a oscilar entre la negación de que haya ningún significado en absoluto en las imágenes, más allá del significado que es colocado ahí por las palabras, y esos otros que reconocen que sí hay (un significado), pero lo consideran como un poder subversivo, en la medida en que agrega alguna cosa a aquello que las palabas le traen bajo la forma de un control. (...) ¿Quién puede entusiasmarse en relación a un documento como el Manifiesto comunista, cuando las transformaciones de la sociedad, para bien o para mal, están ocurriendo a un ritmo tan rápido?".
}

La noción de normalización extraíble de este planteamiento supone una definición del signo icónico que precisaremos aquí. La interpretación del Manifiesto Comunista (soporte papel) como una representación que explota las cualidades de un objeto con menos velocidad que un televisor (formato audiovisual), resulta una interpretación con límites estrechos. Reconociéndolos, se impone que, para equilibrar la eficacia de ambas representaciones, sería suficiente reproducir una versión audiovisual del Manifiesto Comunista. Los soportes tecnológicos que regulan las mediaciones de las cualidades o aspectos del objeto resultan ser, entonces, el libro y la televisión. Con esto, Ransdell argumenta sobre la existencia de una racionalidad que restringe flujos de información para la transformación. Intentaremos llevar este argumento a sus límites. Para interceptar una supuesta razón conservadora, antes que el ejemplo de un televisor y el Manifiesto Comunista, serviría preguntarse por las diferencias entre los efectos de expansión del Manifiesto Comunista y La Riqueza de las Naciones.

Aquí, frente a la comprensión de la ley comunitaria como una restricción deliberada por un sector de la comunidad, oponemos aquella que la asume como un conjunto de reglas de percepción comunes albergadas en las mentes a lo largo de la historia. El proceso de crecimiento de la cadena de mediaciones sígnicas y su dimensión normalizadora remiten ambas una comunidad de pensamiento sin límites claros.

Al avalar seriamente las nociones entrenamiento de habilidades y tecnocinismo, ¿no estaremos, en el intento de proveer libertad al potencial icónico, estableciendo nuevas formas de limitar su poder emancipatorio? Postulamos, después de todo, que en el contexto mismo de las TIC, el anuncio del final de la restricción y la apertura a la libertad no es otra cosa que el enunciado estratégico de un régimen (el de la cultura occidental) que impone el ritmo aceptable del avance tecnológico. 
En este punto, consideramos necesario reponer una visión anti-antropocentrista del asunto. Entendemos que ésta puede reposicionarnos frente a la concepción la mediación evaluadora, entendida por algunos como represión de la creatividad y el cambio.

Martín Serrano (2008: 22) lo había abordado: en las prácticas comunicativas modernas, las conciencias individuales entran en interdependencia. Ahora bien, desde una noción peirceana del sujeto de la mediación (perspectiva no-antropológica) consideramos necesario problematizar uno de las implicancias de la noción modelos de mediación. La comprensión peirceana de la comunidad de pensamiento entiende que los denominados por Martín Serrano (2008) modelos de ajuste son parte constitutiva e ineludibles de la práctica misma de la mediación. Esto es lo que explica el espesor y complejidad de la mediación y es esto lo que, en última instancia, deberá analizarse para dar cuenta de una razón y convención operantes en toda práctica de mediación.

Si una mirada antiantropocentrista es imperiosa para Peirce, es debido a que un agente social no puede diferenciar el límite que separa las necesidades históricas de sus objetivos particulares. El sistema de interdependencias que subsume a la conciencia individual a un medio ambiente comunitario es, además, la fuente rectora de los objetivos individuales (denominada por Martín Serrano [2008] de la lógica del uso funcional). Por esto, proponemos re pensar una noción alternativa a la de "práctica transformadora": la de procesos de cambio.

Identificamos aquí un déficit en la postura de la cubana Nora Gámez Torres (2007). Según la periodista, es posible integrar a la noción serreana de mediación el elemento de sujetos reflexivos actuantes para el cambio social. A continuación, esclarecemos las deudas de este postulado con el enfoque semiótico de la mediación. Esclarecimiento enunciado como sugerencias al desarrollo teórico serreano y a sus intentos actuales de superación.

\subsubsection{Interpretación antiantropocentrista de la ley comunitaria}

Como vimos, un signo con función de mediación, denominado por Peirce representamen, asume relaciones con un fundamento, un objeto y un interpretante. En este punto, nos detendremos en la tercera relación. La mediación sígnica emerge, según el pragmaticismo peirceano, para generar en la mente de alguien más un signo más desarrollado sobre el mismo objeto, es decir, para un interpretante. La función mediadora del signo resulta, así, asociada con los efectos de crecimiento del conocimiento sobre el objeto mediado. Ahora bien, el rumbo de ese trayecto cognitivo creciente es prescripto por el criterio de una comunidad de pensamiento. En otras palabras, una Terceridad (un interpretante) evalúa y, al mismo tiempo, hace avanzar la 
relación siempre evolutiva y cambiante entre las representaciones y los objetos representados. Y Peirce lo hace explícito: lo que él denomina interpretante "encarna la Intermediación (Betweenness) o mediación (...) y alcanza su plenitud en la representación" (Peirce \& Negro Pavón, 1978; 5.104, 1903)

El criterio científico comunitario (interpretante), al normalizar las relaciones sígnicas, las habita y las dispone a la llegada de un nuevo signo, inminente, que a su vez las normalizará. Peirce (en Saleta de Salvador, 2010: 9; CP 1.339) ilustra esto categóricamente: "al final, el interpretante es tan sólo otro signo al cual se entrega la antorcha de la verdad; y en calidad de signo tiene a su vez su interpretante. He aquí una serie infinita".

Subrayamos aquí el papel que la noción interpretante puede desempeñar en la comprensión del proceso de mediación. El Interpretante entraña, en la cadena de signos, una operación simbólica que consiste ponerse en relación con otra relación. Representa, desde que es generado, la relación de envío entre un signo y un objeto. La cadena de la mediación sígnica queda ilustrada en un sistema de reenvíos, en el que el significado de una mediación vendrá dado por la práctica mediadora generada por ella. Peirce (en Andacht, 2003: 225) es lo suficientemente claro al asegurar que "un símbolo es un signo que significa eso que será interpretado que significa".

Con todo, un interpretante no es un acto de una voluntad individual, capaz de desviar un flujo de sentido. Es, en cambio, un tipo de signo que, por un lado, otorga un significado convencional $y$, por el otro, espera ser desarrollado por un nuevo signo. En esta definición no cabe la idea del agente-actor del cambio, pero sí, como hemos intentado expresarlo hasta aquí, la de un sujeto colectivo de evolución histórica. Sujeto construido en el proceso de incremento de conocimiento del mundo. Parece necesario asumir la distancia que separa una instancia lógica como el interpretante de una agencia de interpretación. Sara Barrena (2006: 116) es clara al advertir que "de acuerdo con el pragmaticismo peirceano, un estudio de la creatividad no puede detenerse en el sujeto, sino que debe fijarse en los frutos, en cómo se produce el resultado de la acción, y la obra creativa aparece, según Peirce, a través de la abducción". La abducción, como la define Peirce (\& Negro Pavón, 1978; CP 5.194, 1903) es la operación por la cual se introduce una novedad que aumenta la inteligibilidad del mundo. Es en la abducción donde surge (y no donde se agencia) una conjetura nueva.

\subsubsection{La ley y los intérpretes}

Para entender lo simbólico de la mediación, es útil tener presente, como lo asegura Debrock (1991: 54), que "el punto capital acerca de un signo no es tanto que esté por algo, como que sea reconocido como algo que está por algún otro". 
Entonces, es a partir de la postulación de un sujeto histórico de conocimiento y de una mediación sígnica que está en función de él que podemos entender el funcionamiento histórico de la cadena de signos. Y, a la vez, es desde aquí que nos será posible asimilar las posibilidades de reorientar de las convenciones comunitarias que habitan las prácticas de mediación. El interpretante, asumido por el peirceanismo constructivista como regulación impiadosa del potencial icónico, merece entonces una observación. Las mutaciones son producidas en tiempos largos y el cambio debe ser pensado como conjunto de procesos agenciados por un sujeto complejo, sin anclaje en el individuo sino en el espacio donde el individuo inscribe su presencia.

\subsection{4. ¿̇nterpretante o intérprete?}

Arribamos, por esta ruta, a distinguir el interpretante del intérprete. Si la instancia lógica del interpretante no es agenciada por un intérprete, la función de mediación sígnica no se explica finalmente por un individuo mediador. Eliseo Verón (1981), al referirse a la red de signos producida en la semiosis, afirma que en el desarrollo de la cadena de comunicación, cada cuerpo actuante se disuelve en su univocidad orgánica para constituir el lugar de paso del sentido. Se produce, en sus palabras, "una desagregación de la red de los cuerpos actuantes" y se conforma un "espacio multidimensional".

La fuente de este espacio no está en la palabra pronunciada por una persona, sino en las relaciones de contigüidad o metonimia a la que esa palabra reenvía. En otras palabras, uno no piensa en la soledad de su corazón, sino con operaciones simbólicas aprehendidas en su inserción cultural. Las reflexiones de Verón, y ante todo sus definiciones premetodológicas, se inscriben en una corriente de la semiótica que recupera dos elementos que entiende esenciales del pragmatismo peirceano: la continuidad infinita de la cadena de signos y la representación como una operación simbólica de reenvío.

\subsubsection{Eliseo Verón: el descentramiento y la interacción discursiva}

Eliseo Verón estuvo a cargo de la primera traducción de Antropología estructural (Lévi-Strauss, 1979) al español. Su propuesta se inscribe en la serie de postulados teórico-analíticos con una posición orientada al descentramiento de los procesos de sentido. El autor toma influencias de la perspectiva nietzscheana del no-centro (del sin-origen, sin-verdad). La afirmación nietzscheana ofrece albergue a nuevas hipótesis de la significación que la desvinculan de un fundamento y de un cuerpo actuante y la integran al funcionamiento de las prácticas sociales de mediación: "iY no digamos el yo! Ha pasado a ser una fábula, una ficción, un juego de palabras" (Nietzsche, 1998: 
22). A favor de la noción de la contingencia y de la inocencia del devenir, la afirmación nietzscheana nos introduce a la preocupación por el funcionamiento de los procesos de mediación en relación con el tiempo. Tales procesos, dirá Verón (1997), se dan en un contexto de relaciones de contigüidad entre distintas representaciones en las que la unidad material de la palabra del hombre se desintegra a la vez que se integran colectivos de identificación. Nuevas unidades, no-orgánicas, donde convergen las operaciones simbólicas de mediación.

Al postular la formación de colectivos de identificación en los procesos de semiosis, Verón no hace otra cosa que reformular la relación dialéctica asignada por Martín Serrano (2008) entre acciones intencionales, prácticas institucionalizadas y constricciones sociales. Sin embargo, el acento es trasladado por Verón de la interacción entre los tres elementos a las zonas constituidas en los espacios simbólicos creados entre ellos. El autor argentino propone la noción zonas de formación colectivos de identificación para ilustrar un aspecto ya relevado, por ese entonces, por el postestructuralismo: el carácter agonístico de los espacios semióticos. Los colectivos de identificación a los que las prácticas de mediación remiten son formados en la interacción conflictiva entre prácticas discursivas, en un campo enunciativo dinámico. Volviendo, desde este punto, a Peirce, el efecto de identificación que la comunidad de pensamiento puede producir en el sujeto de discurso no es guiado por el individuo, sino por una comunidad de pensamiento productora tanto de la identificación como de la distinción y la oposición.

Cabe, así, relativizar la afirmación de Gámez Torres (2007) de que los efectos de control de las operaciones simbólicas deben ser abordadas en el contexto de apropiación de éstas por el individuo. Es desanclando el problema de sus límites materiales e individuales que advertiremos las estrategias de control reproducidas en la misma práctica de la mediación. Esta es la línea que sigue el estudio de la mediación en América Latina, donde el problema que interesó a Martín Serrano es leído en sede Semiótica (Escudero, 1997; 2008; Fernández, 2010; 2011) a partir de enfoques generales provistos por el mismo Peirce, Angenot (2010), Verón (1981; 1997), Thompson (2003) y Hjarvard (2013), y con una mirada sociológica de importante influencia postestructuralista francófona (Derridá, 1989; Foucault, 2008; 2011). Un proyecto teórico-analítico interesado en cristalizar las condiciones de producción de colectivos de identificación; en abordar, por lo tanto, la mediación como la operación que actualiza los límites entre esos colectivos. En fin, en compartir, en este punto, el ámbito en el que van Dijk $(2000,2012)$ ha situado sus trabajos: la interacción discursiva.

Desde aquí, es necesario revisar el principio de que la mediación estructural apresa los acontecimientos en formas comunicativas y reajusta el desfasaje producido por el cambio social que erosiona los modelos de mediación (Martín Serrano, 2008). Los 
modelos de mediación, antes bien, son aplicados reajustando los desfasajes que ellos mismos promueven continuamente. Lo que subrayan Peirce y los autores de la semiótica post-estructuralista en los que el pragmatismo tuvo resonancia es, precisamente, el dinamismo de los campos enunciativos. No hay modelos rígidos que restrinjan el cambio. Hay, en efecto, modelos en constante proceso de reconstrucción simbólica.

Si insistimos en esta dirección es porque direcciones alternativas conducirían a la ilusión de alcanzar un punto cero del sentido, en el que el poder normalizador de un modelo de mediación neutralice la erosión del cambio o en el que el cambio conmueva completamente los modelos rendidos ante la persistencia de su acción. A nuestro entender, lo que pierde vigencia y se desactiva es la problemática de la mediación planteada por Martín Serrano (2008) como reajuste del desfasaje entre reproducción y cambio social.

\section{LA LEY Y LA VERDAD}

Lo observado hasta ahora sobre los procesos simultáneos de normalización y dinamización de los campos enunciativos adquiere, precisamente por dicha simultaneidad, una utilidad doble. Por un lado, hemos subrayado la existencia de un régimen de enunciación actuante y, por otro lado, nos hemos encaminado a describir los efectos de verdad producidos en el fenómeno de la mediación sígnica.

\subsection{Regímenes de enunciación}

En cuanto al régimen de enunciación que prescribe las modalidades enunciativas en los medios, la comunicación interpersonal y las instituciones del Estado, apuntaremos algunos elementos sugeridos por la pragmática peirceana.

La noción semiótica no-constructivista de la mediación sostiene que existen creencias fijadas en los agentes sociales de una comunidad. Su expectativa de sentido es la que orienta la generación de interpretantes, es decir, de futuros signos que re interpretarán su misma representación del mundo. Para Peirce (\& Zenón González, 2002; CP 1.212, 1902), la mediación es análoga al ejercicio de apuntar al blanco con un rifle, en el cual se observa la influencia del futuro en el presente mediante la acción de la mente que anticipa el objetivo y en base a éste imparte direccionalidad a la fuerza física, ciega y compulsiva del mundo.

Una vasta cadena de interpretantes significan y re significan las representaciones de diversos aspectos de la experiencia que aún siguen asegurando el conocimiento de un acotado perímetro. Así, el ajuste a la realidad pura y total se debate en sus repre- 
sentaciones, que confirman que el recuerdo de la experiencia aguarda el significado que será otorgado en el devenir de recuerdos posteriores. Pero entonces, ¿cuál es el lugar de las convenciones comunitarias en esa cadena de representaciones? ¿Las convenciones remiten a un acuerdo pasado o a una construcción en devenir?

\subsubsection{La ley y la evolución del sentido}

En este punto, es útil recordar el concepto de sinequismo. La noción remite a una serie de tres principios cosmológicos que Peirce (\& Vericat, 1988; CP 6.169, 1892) postula para decir, en resumidas cuentas, que existe en el universo una tendencia a instaurar leyes. El sinequismo remite al principio de continuidad que establece que el significado asignado por un interpretante a un signo será reformulado por un nuevo interpretante.

En este marco, la incapacidad de innovación del sí mismo, el self (Andacht, 2008), plantea un nuevo interrogante: ¿¿Debemos hablar de la represión de la ley sobre el potencial de conocimientos del mundo o de una mentalidad individual no-consciente con habilidades perceptuales aprendidas en un marco comunitario normalizador, pero en evolución? Aquí resuena las premisas de Verón (1997) sobre la producción de colectivos de identificación y de transferencias de marcos interpretativos (1981). No es una ley opresora la que delimita ese potencial, sino la sucesiva insuficiencia de los signos para significar la experiencia la fuerza que alienta desde el devenir (como un blanco de rifle) la representación de algunos respectos del objeto y la exclusión de otros.

Umberto Eco (1992) desliza una reflexión en conexión con esta cuestión. La verdadera noción de realidad envuelve una noción de comunidad creando un significado intersubjetivo. Asimilar el espesor de esta afirmación de principio exige, como requisito, comprender que la relación entre ley y evolución no siempre es de tensión sino de generación.

En contraste con la visión no-constructivista expuesta, se distingue la esgrimida por Da Silveira (2004: 75) sobre las posibilidades de creatividad del individuo. Según el autor, para percibir los fenómenos de la realidad y representarlos, "se supone que los admiremos y nos dejemos ser por ellos atraídos". Esto supone, a las claras, la posibilidad de un individuo inteligente que, luego de restringir una potencialidad, decide liberarla y otorgarle su capacidad de decisión. Lo naturalmente admirable de los fenómenos que da Silveira da por sentado vería habilitado su tránsito libre a las representaciones de los individuos. La presunción de da Silveira es sobre una mentalidad que se entrega a ser atraída por los hechos. Pero, como está a la vista, la ficción de un 
individuo que se permite ser atraído por un objeto de deseo implica la realidad de uno que se preserva conscientemente la potestad de prohibirlo.

La concepción antropocéntrica concluye que la creatividad individual supera el umbral de las habilidades perceptuales comunitarias y conserva la facultad privilegiada de introducir novedades. Con esto, el sujeto individual consciente sería capaz no sólo de abstraerse del flujo de mediaciones-signos que desarrollan el conocimiento del objeto mediado. Podría, además, clasificar unas mediaciones como antiguas y otras, los propias, como novedosas. Son didácticas, en este punto, las instrucciones de Peirce (\& Restrepo, 2003; CP 2.228, c.1897) sobre lo diacrónico de la sucesión de mediaciones. El pensamiento, según el lógico norteamericano, se produce en un instante de tiempo imposible de percibir inmediatamente. En ese instante no hay duración. Por lo tanto, el pensamiento, al próximo instante, habrá sido sustituido por otro que lo interpretará y se dispondrá él mismo a una nueva interpretación. Pensamos en el tiempo pero nos resulta imposible percibir inmediatamente su transcurso. En tal caso, debería existir una percepción de lo temporal a cada instante. Sin embargo, en un instante no hay duración y, por lo tanto, tampoco una percepción inmediata de la duración.

En otras palabras, ¿cómo es posible que el sujeto que se encuentra en el pensamiento, y no a la inversa, perciba lo viejo y la necesidad de lo nuevo e impulse un acto concreto de transformación si no es consciente de la duración de sí mismo en ese instante de tiempo? Aquí damos en una cuestión elemental de la mediación comúnmente soslayada.

Nuestra pregunta señala la necesidad de revisar la perspectiva construccionista y psicologista de la mediación y sugiere una nueva pregunta: ¿en qué lugar se ubica la Terceridad normalizadora que aplica, sobre las posibles representaciones, la ley de las representaciones aceptables?

\subsubsection{Procedimientos negativos}

Recapitulando, la regulación del cambio debe ser considerada como parte de un sistema o régimen regulatorio en evolución. Sin embargo, en ese trayecto evolutivo operan procedimientos negativos sobre la mediación-sígnica, que delimitan unos aspectos del objeto y dejan otros en potencia. Ransdell (2005), en este sentido, considera que toda Terceridad actúa sintetizando los factores en la percepción, lo que hace que sea una percepción de un objeto antes que de otro, y de unos de sus aspectos en lugar de otros. El acto positivo de mediación es producido simultáneamente al acto negativo de escansión y delimitación. 
Verón (1985), en Semiosis de lo ideológico y del poder, echa luz sobre el carácter estratégico de esas escansiones y cortes, y Debrock (1991), al proponer la categoría de eventos de signos y afirmar que la representación es algo que sucede, reconoce la puesta en función de mecanismos de control y exclusión: "un evento elimina irrevocablemente algunas posibilidades y deja ser a otras" (Debrock, 1991: 53). Andacht (2008), por su parte, se pregunta por los regímenes simbólicos de vigilancia y castigo.

En síntesis, a lo largo del proceso de significación desplegado en los eventos de mediación, los aspectos no conocidos del mundo son confinados por una racionalidad comunitaria al terreno de cualidades cognoscibles. La verdad absoluta y completa es imaginable únicamente en su aplazamiento.

\subsection{La verdad en su postergación}

El despliegue de la cadena de mediaciones produce, en el sentido que aquí le asignamos, efectos de verdad. El no-constructivismo ha establecido claramente su punto de vista sobre esto. La potencialidad de lo por conocer del objeto no está las cualidades de las cosas, sino en el devenir de una razón normalizadora que indica lo cognoscible. Peirce (\& Barrena, 2005; CP 2.293, 1903) designa a esa razón como interpretante inmediato ("ley o regularidad del futuro indefinido"). La significación completa o la verdad del objeto es, en el transcurrir de la cadena de signos, una instancia sucesivamente postergada. A esto hace referencia Shapiro (2002: 123) al asegurar que "este proceso es necesariamente siempre histórico y no dado a priori". Así, entender la verdad histórica es asumir su conformación en pleno desarrollo.

\subsubsection{La mediación. Acto presente y programático}

Pero entonces, la historia actúa por medio de la acción de signos, de eventossignos (Debrock, 1991). Es de interés preguntarnos por la imagen que los signos nos devuelven de la historia. ¿Cómo operan los signos a los que atribuimos la representación de los hechos producidos en el tiempo?

Ransdell (1997: 61) repara en este asunto con un enfoque no-constructivista y nos ofrece una primera respuesta: "en rigor, no percibimos nada a través de nuestros sentidos pero el mero hecho que el objeto es en el pasado, no puede, él mismo, ser una objeción para decir que el recuerdo verídico es una percepción directa del objeto recordado". Vale detenerse aquí.

Según esta premisa, parece claro que la memoria no es una inferencia a partir de la observación del hecho fáctico. Desarrolla, en cambio, el conocimiento que las sucesivas representaciones conmemorativas previas habían transmitido sobre él. Esta 
conclusión parece ir de suyo, pero hace alusión a la función programática de las mediaciones sígnicas en la cadena de signos. Decir, como lo hace Debrock (1991), que las condiciones del "evento-signo" fueron dispuestas por un evento-signo anterior es equivalente a decir que la mediación es un fenómeno del presente que establecerá las condiciones para una mediación futura. Así, la verdad de los hechos y de los objetos del mundo está dada por la verdad acordada entre las representaciones que los significarán hasta un "futuro indefinido" (Peirce \& Barrena, 2005; CP 2.293, 1903). En palabras del mismo Peirce (\& Serra; CP 7.361, 1873) "el pensamiento sólo es racional en la medida que se propone para un posible pensamiento futuro".

\subsubsection{Proceso de sentido y proceso de cambio}

Es necesario sopesar las consecuencias de este razonamiento. Es aquí donde encontramos una clave de lectura para asumir la posibilidad del cambio. No hay ruinas que retengan lo fáctico del hecho. La mediación no apresa ni captura aspectos del objeto mediado. Las ruinas de un edificio romano no re instalan su pasado, sino la novedad de la representación actual de algunas de sus cualidades. La mediación es una actualización del sentido de un objeto, no la actualización del objeto.

El evento de la mediación interpreta nuevas cualidades del objeto, no representadas hasta el momento. La posibilidad de lo nuevo aquí aparece atada al desplazamiento de la cadena de signos sobre el tiempo. A la luz de esto, no resulta complejo comprender la reflexión de Michel Pollak (2006) sobre los testimonio de experiencias extremas. Según Pollak (2006: 53), existen "principios generadores y organizadores de las prácticas y de las representaciones" y todo testimonio es una reconstrucción inconsciente de la propia identidad. Ésta, según el autor, es producida por testimonios normalizados en el espacio de lo decible. Los testimonios que re-significarán el pasado están en el futuro de mi testimonio. Son la posibilidad de la novedad pero, al mismo tiempo, se pronuncian dentro de límites simbólicos y regímenes enunciativos.

Esta es la óptica adoptada por Eco (1992) cuando designa una pasión trascendental. Tal pasión, entiende, evoca la existencia de una comunidad como garante intersubjetiva de la verdad ${ }^{2}$.

En consonancia, Andacht (2003) propone que existen ciertas fórmulas comunitariamente acuñadas de lo aceptable, actuantes en aquello que denomina aids (implementos de ayuda). No aluden a ayudas solicitadas, ni voluntariamente procuradas,

2 Umberto Eco (1992) plantea que la verdadera noción de realidad envuelve una noción de comunidad creando un significado intersubjetivo. En consonancia, Ransdell (1997: 25) nos recuerda que "la teoría de Peirce es una descendencia lógica de Kant". 
sino a ayudas-instrucciones disponibles en una matriz que orienta las representaciones según marcos interpretativos comunes.

En sede sociológica, Maurice Halbwachs (2004) ya había reflexionado en los términos de Ransdell y Andacht. En La mémoire collective: cadres sociaux de la mémoire, el sociólogo considera que el individuo no recuerda solo, sino con la ayuda de los recuerdos de otros. Paul Ricoeur (2000: 126) lo retoma en estos términos:

"uno de los aspectos principales quizá consista en que nuestros recuerdos se encuentran inscritos en relatos colectivos que, a su vez, son reforzados mediante conmemoraciones y celebraciones públicas de los acontecimientos destacados de los que dependió el curso de la historia de los grupos a los que pertenecemos".

\subsubsection{Precaria verdad}

Como vimos, y nos recuerda Ransdell (1997: 28), "lo que un signo genera es otro signo, cualquier cosa que puede jugar el rol del interpretante en una actualización". El ser de la mediación que actualiza la identidad del objeto es un "ser" a completarse en la sucesión de representaciones. Diremos aquí, por lo tanto, que la significación verdadera de un objeto habita en cada una de sus postergaciones.

En la misma dirección, Shapiro (2002: 19) afirma que "todos los interpretantes dinámicos (la mediación última posible) están a la altura de la verdad plena sobre el objeto" y que ésta "es un ideal que podría ser aproximado pero nunca alcanzado". El transcurrir del tiempo y el trayecto positivo de mediaciones resuelven parcialmente la carencia de verdad de las representaciones pero no resuelven la precariedad producida por las nuevas ausencias y las correspondientes expectativas. "No hay signos a menos que haya expectativas", nos confirma Debrock (1991: 60). El único a priori que precede una mediación determinándola está fundado en la posibilidad y se encuentra en el devenir del significado.

Con todo, abordar la problemática del cambio a la luz de sus relaciones con la posibilidad de verdad y con la acción de la ley sólo es posible, desde una perspectiva peirceana no-constructivista.

\section{LAS PALABRAS Y LAS COSAS}

Finalmente, proponemos una comprensión específica del lugar que ocupa el cambio en el fenómeno de la mediación sígnica. Peirce no consagra sus estudios al campo de la lingüística, pero se refiere, como vimos, a las relaciones convencionales establecidas en la representación de la palabra: "la comunidad aprueba una cierta forma arbitraria de relacionar, por remisión, las palabras y los objetos" (Peirce \& Barrena, 
2005; CP 2.280, 1903). Esta observación implica una comprensión particular del eje estabilidad/cambio que aquí nos interesa. La relación que establece entre las palabras y las cosas es de un tipo específico: de remisión. Es decir, los signos nos reenvían a los objetos. Aquí encontramos una noción de continuidad que exige pensar en el tiempo de la cadena de signos de mediación.

Si una serie de mediaciones resulta una sucesión de reenvíos es por la acción del tiempo. Las relaciones entre mediaciones sígnicas son puestas en función e impulsan, según el pragmatismo, a consecuencias prácticas. El contexto de las consecuencias prácticas de las mediaciones será el mismo que el de las mediaciones mismas: un proceso diacrónico, de crecimiento del sentido. Allí es donde se producen, entonces, una secuencia de movimientos de continuidad-progresión por los cuales no todo lo que dice un signo sobre un objeto es equivalente a lo ya dicho por signos anteriores. Por lo tanto, el cambio no es sólo admitido el pragmaticismo peirceano sino incluido a la noción misma de progresión de los procesos de sentido. Los procesos de sentido son, por su dinamismo, procesos de cambio.

\subsection{El cambio en el sistema de la lengua}

La lingüística estructural, en su definición del vínculo entre lengua y lenguaje, había atribuido al cambio un lugar marginal. Saussure (1945) entendía que la lengua es un sistema de correspondencias por oposición que se mantiene inmutable. Aquí, el tiempo no cumple otra función que la de garantizar el resguardo del sistema de la lengua y el sujeto hablante la de custodiar inconscientemente su estabilidad.

En un plano, el diacrónico, se registran cambios, reajustes, nacimiento de nuevos dialectos, etc.; mientras que en otro, el sincrónico, sólo importa el estado de la lengua, que organiza de una forma estable los elementos del sistema. Saussure focaliza en el estudio del sistema de la lengua y de las oposiciones irreductibles contraídas en sus límites, como significado-significante, individuo-sociedad o lengua-lenguaje. Sostiene, así, que los cambios fonéticos introducidos por los hablantes de una lengua sólo persisten en el sistema como efectos ruinosos. Los conceptos de cambio y evolución remiten a hechos diacrónicos que no guardan relación con los estados de la lengua (hechos sincrónicos). Éstos sólo conservan, en estado ruinoso, los efectos escurridizos del cambio. El mismo Saussure (1945: 115) lo confirma al considerar que "es evidente que el aspecto sincrónico prevalece sobre el otro (...) pues si el lingüista se sitúa en la perspectiva diacrónica, no será la lengua lo que perciba, sino una seria de acontecimientos que la modifican". 


\subsection{El cambio en el discurso}

Verón (1997), en sus reflexiones sobre la producción del mensaje mediático, aporta a esta discusión sobre la mediación y el cambio. El autor argentino propone pensar, mediante la categoría mediatización, en la naturaleza de los procesos de cambio social del post modernismo. "No hay procesos lineales entre una causa y un efecto; nos encontramos frente a una maraña de circuitos de "feedback»" (Verón, 1997: 13). En este marco, el autor asegura que en el seno de la comunicación se producen colectivos de identificación en tanto construcciones. Él mismo anuncia su sede al decir que la noción de " "colectivo» remite (...) al concepto de «interpretante» en la semiótica de Peirce y es un aspecto central del funcionamiento de las estrategias enunciativas" (Verón, 1997: 14). Un tema central en el análisis veroneano de los procesos de mediatización es que estos colectivos de identificación producen cuadros identitarios que agrupan a actores individuales. Según Verón, son los permanentes reacomodamientos de las zonas de producción de estos colectivos los que caracterizan nuestros tiempos: el cambio incluido al proceso de sentido. Esta definición esboza el concepto de la entidad asignada a la transformación. Ésta no es otra cosa que el efecto de conjunto de los procesos de sentido desarrollados en la comunicación de nuestros días. En definitiva, "el término mediatización no signa otra cosa que lo que es hoy el cambio social de las sociedades post-industriales" (Verón, 1997: 16).

\section{CONCLUSIÓN}

La percepción y la mediación sígnica que ella produce, en conclusión, dejan de ser del individuo desde que adopta el criticismo moderado adquirido en su colectivo de identificación, donde aprende las habilidades para la definición de la verdad, la libertad y sus contrarios.

La afirmación referida a la producción de un evento-signo (Debrock, 1991) supone, por un lado, que las zonas de producción de colectivos de identificación (Verón, 1997) son dinámicas. Pero, por otro lado, que los fenómenos de la realidad, portadores de una fuerza ciega que nos dirige la atención a algún respecto del objeto (Peirce \& Barrena, 2005; CP 2.306, 1903), limitan la introducción de lo totalmente inédito. Lo real, como una fuerza ciega y punzante -la Segundidad, según Peirce (\& Barrena, 1997; CP 6.452-485)-, interpela nuestra habilidad perceptual inmediata $y$, como lo hemos subrayado, mediada.

Con todo, la ley, representación de lo universal, no es una fuerza implacable que recae sobre la percepción, sino una fuerza "localizada, como poderes activos o pasivos" (Ransdell, 1997: 36), en las mismas mediaciones-sígnicas. Las convenciones que 
erigen las leyes aparecen albergadas en las mismas representaciones. Les otorgan simultáneamente dinamismo comunicativo y regularidad.

Los límites impuestos a la novedad y el cambio tienen su origen en el carácter normalizador de toda mediación. Cualidad, ésta, tan propia de las mediaciones como la de su potencial transformador. La polémica abierta en torno a la facultad de creación de las mediaciones debería someterse a una premisa que se desprende de nuestra reflexión: la creación y el cambio no emergen como el fenómeno de lo insólito e inédito, sino como el de la producción de diferencias en relación con un trayecto histórico de mediaciones. Un fenómeno ocurrido como un efecto de conjunto en el espacio de la experiencia comunitaria.

\section{BIBLIOGRAFÍA}

ANDACHT, F. (2003): "Joseph Ransdell entrevistado por F. Andacht", De signis, n 4, pp. 221234.

ANDACHT, F. (2008): "Self y creatividad en el pragmatismo de C. S. Peirce: «la incidencia del instante presente en la conducta»", Utopía y Praxis Latinoamericana, vol. 12, n 40, pp. 3965. Disponible en http://goo.gl/UIMGRL. Consultado el 14 de noviembre de 2014.

ANDACHT, F. (2013): "Análisis de un episodio de la miniserie cidade dos homens como una fábula indicial", Situarte, n 14, pp. 9-19.

ANGENOT, M. (2010): El discurso social. Los límites históricos de lo pensable y lo decible. Buenos Aires: Siglo XXI.

BAJTín, M. (1982): Estética de la creación verbal. Buenos Aires: Siglo XXI.

BARRENA, S. (2006): "La creatividad en Charles S. Peirce", Anthropos, n² 212, pp. 112-120.

BARRENA, S. \& NUBIOLA, J. (2006): "Antropología pragmatista: el ser humano como signo en crecimiento", II Jornadas "Peirce en Argentina", 7-8 de septiembre del 2006, Buenos Aires.

COLAPIETRO, V. (1995): "Notes for a Sketch of a Peircean Theory of the Unconscious", Transactions of the Charles S. Peirce Society, vol. 31, n³, pp. 482-506.

DA SILVEIRA, B. (2004): "Observe-se o fenómeno: forma e realdade na semiótica de Peirce", Cognitio, vol. 5, n² 2, pp. 72-77.

DEBROCK, G. (1991): "La información y el estatuto metafísico de los signos”, Comunicación y Sociedad, vol. 4, n 1-2, pp. 53-64. Disponible en http://goo.gl/70Bqbz. Consultado el 10 de noviembre de 2014.

DERRIDA, J. (1989): La escritura y la diferencia. Barcelona: Anthropos.

ECO, U. (1992): Los límites de la interpretación. Barcelona: Lumen. 
ESCUDERO CHAUVEL, L. (1997): "The media contract", in Winfried, N. (ed.): Semiotics of the media. State for the art, projects and perspectives. Berlín: Mouton de Gruyter, pp. 99-107.

ESCUDERO CHAUVEL, L. (2008):"Espacio público y espacio de los medios: la agenda y las elecciones mexicanas", Cuadernos de Información y Comunicación, n²13, pp. 107-130.

FERNÁNDEZ, J. L. (2010): "La mediatizacion del sonido y la vida musical", Chasqui, n 15, pp. 38-41.

FERNÁNDEZ, J. L. (2011): "Medios de sonido y vida social”, Letra, Imagen, Sonido. Ciudad Mediatizada, $\mathrm{n}^{\circ}$ 6-7, pp. 71-80.

GÁMEZ TORRES, N. (2007): “El paradigma de la mediación: crítica y perspectivas", Mediaciones Sociales, № 1, pp. 195-213. Disponible en http://goo.gl/77BiiC. Consultado el 27 de junio de 2015.

HALBWACHS, M. (2004): La mémoire collective: Los marcos sociales de la memoria. Barcelona: Anthropos.

HJARVARD, S. (2013): The mediatization of culture and society. New York: Routledge.

LÉVIS-STRAUSS, C. (1979): Antropología estructural: mito, sociedad, humanidades. Madrid: Siglo XXI.

MARTÍN SERRANO, M. (2008): La mediación social. Madrid: Akal.

NIETZSCHE, F. (1998): El ocaso de los ídolos. Buenos Aires: Tusquets.

PEIRCE, C. \& BARRENA, S. (1997 [1908]): Un argumento olvidado a favor de la realidad de Dios. Publicado en CP 6.452-485. Disponible en http://goo.gl/JgmGyc. Consultado el 12 de noviembre de 2014.

PEIRCE, C. S. \& BARRENA, S. (2005 [1903]): El ícono, el índice y el símbolo. Publicado en 2.274308. Disponible en http://goo.gl/KyFDGW. Consultado el 12 de noviembre de 2014.

PEIRCE, C. \& NEGRO PAVÓN, D. (1978 [1903]): La realidad de la terceridad. Publicado en CP 5. 93-119. Disponible en http://goo.gl/exYQLV. Consultado el 5 de julio de 2015.

PEIRCE, C. S. \& RESTREPO, M. (2003 [1897]): Fundamento, objeto e interpretante. Publicado en CP 2.227-229. Disponible en http://goo.gl/QZRcRv. Consultado el 13 de noviembre de 2014.

PEIRCE, C. S. \& SERRA, J. P. (2007 [1873]): Que la significación del pensamiento reside en su referencia al futuro. Publicado en CP 7.358-361. Disponible en http://goo.gl/UjDKMi. Consultado el 12 de noviembre de 2014.

PEIRCE, C. \& VERICAT, J. (1988 [1868]): Algunas consecuencias de cuatro incapacidades. Publicado en CP 5.264-317. Disponible en http://goo.gl/auHXmj. Consultado el 12 de noviembre de 2014.

PEIRCE, C. S. \& VERICAT, J. (1988 [1892]): La ley de la mente. Publicado en CP. 6.102-394. Disponible en http://goo.gl/LeCZF8. Consultado el 12 de noviembre de 2014. 
PEIRCE, C. S. \& ZENÓN GONZÁLEZ, J. (2002 [1902]): Acerca de la ciencia y las clases naturales. Publicado en CP I. 203-237. Disponible en http://goo.gl/t1qd0x. Consultado el 2 de julio de 2015.

PIAGET, J. (2000): El nacimiento de la inteligencia en el niño. Barcelona: Crítica.

POLLAK, M. (2006): Memoria, olvido silencio. La producción social de identidades frente a las situaciones límites. La Plata: Al Margen.

RANSDELL, J. (1977): "Some Leading ideas of Peirce's semiotic", Semiótica, vol. 19, n³-4, pp. 157-178. Disponible en http://goo.gl/bmOEX. Consultado el 13 de noviembre de 2014.

RANSDELL, J. (1997): On Peirce's conception of the iconic Sign. Versión 2.0. Texas: Dept. of Philosophy, TexasTechUniversity. Disponible en http://goo.gl/3ugh5. Consultado el 13 de noviembre de 2014.

RANSDELL, J. (2005): The epistemic function of iconicity in perception. Version 2.0. Texas: Dept. of Philosophy, Texas Tech University. Disponible en http://goo.gl/4kYkV7. Consultado el 28 de junio de 2015.

RICOEUR, P. (2000): La memoria, la historia, el olvido. Buenos Aires: Fondo de Cultura Económica.

SALETA DE SALVADOR, A. (2010): "La «semiosis ilimitada» del hipertexto como texto en acción", en Hynes, C. (ed.): IV Jornadas GEP Argentina: 26-27.08.10. Buenos Aires: Grupo de Estudios Peirceanos en Argentina. Disponible en http://goo.gl/WYnHqA. Consultado el 2 de julio de 2015.

SAUSSURE, F. de. (1945): Curso de lingüística general. Buenos Aires: Losada.

SHAPIRO, M. (2002): "Aspects of a Neo-Peircean Linguistics: Language History as Linguistic Theory", en Shapiro, M. (ed.): The Peirce Seminar Papers. Essays in Semiotic Analysis. Vol. V. NY/Oxford: Berghahn Books, pp. 108-126.

THOMPSON, J. B. (2003): "La transformación de la visibilidad", Estudios Públicos, n 90, pp. 273-296. Disponible en http://goo.gl/wop5Qz. Consultado el 30 de junio de 2015.

VAN DIJK, T. A. (2000): Estudios del discurso: introducción multidisciplinaria. Vol. 2, El discurso como interacción social. Barcelona: Gedisa.

VAN DIJK, T. A. (2012): Discurso y contexto. Un enfoque sociocognitivo. Barcelona: Gedisa.

VERÓN, E. (1981): La semiosis social. Barcelona: Gedisa.

VERÓN, E. (1985): "Semiosis de lo ideológico y del poder", Contratexto, n 1, pp. 11-30.

VERÓN, E. (1997): "Esquema para el análisis de la mediatización", Diálogos de la Comunicación, $\mathrm{n}^{\circ} 48$, pp. 9-16. 
Paulo Damián ANICETo es Licenciado en Comunicación Social por el Colegio Universitario de Periodismo Obispo Trejo y Sanabria. Doctorando en Socio-semiótica del Centro de Estudios Avanzados de esa institución. Integrante del Programa de investigación Estudios sobre la Memoria del Centro de Estudios Avanzados de la Universidad Nacional de Córdoba, Argentina. Autor de ensayos teóricos, como "La memoria como un signo de deudas impagas" y "El factor histórico de la lengua y el tiempo de los discursos", publicados en las revistas especializadas Question, de la ciudad de La Plata, y Forma Función, de la ciudad de Bogotá. Autor del trabajo de investigación semiótica titulado "ESMA, centro para la memoria mítica e histórica. Estudio sobre las memorias que recuerdan o cierran el pasado de la última dictadura militar argentina", publicado en Fecundidad de la memoria. Desafíos del presente a los usos del pasado en América Latina. Correo electrónico: paulodamiananiceto@gmail.com. 